\title{
INTERACCIÓN Y ACCESIBILIDAD PARA UNA EDUCACIÓN INCLUSIVA: UN ENFOQUE DE COMPUTACIÓN UBICUA Y LINGÜÍSTICA COMPUTACIONAL
}

\author{
INTERACTION AND ACCESSIBILITY TO INCLUSIVE EDUCATION: AN APPROACH \\ TO UBIQUITOUS COMPUTING AND COMPUTATIONAL LINGUISTICS
}

Janio Jadán-Guerrero ${ }^{1,3}$

Luis A. Guerrero ${ }^{1}$

María A. Carpio-Brenes ${ }^{2}$

\begin{abstract}
RESUMEN
El desarrollo de tecnologías inalámbricas y dispositivos computacionales móviles abren la posibilidad de nuevos escenarios de aplicación en el campo de la lingüística computacional. Uno de ellos es la integración de estudiantes con necesidades educativas especiales a escenarios de aprendizaje interactivos y accesibles. El artículo describe un sistema ubicuo para fortalecer la interacción en el proceso de lectura inicial de estudiantes con síndrome de Down. El sistema está basado en el método de lectoescritura Picto Fónico (PiFo), al cual se incorpora una interfaz tangible compuesta por objetos físicos y letras tangibles. Estos objetos pueden ser reconocidos por Pifo, un oso perezoso de peluche que tiene incorporada tecnología RFID (Identificador de Radio Frecuencia).

En el Centro de Educación Especial Centeno Güell de Costa Rica se diseñó una fase experimental para evaluar aspectos de interacción mediante actividades para el desarrollo de la conciencia fonológica en el proceso de lectura inicial. Participaron 4 maestras y 6 estudiantes con síndrome de Down. Los resultados obtenidos permitieron evidenciar la estimulación inmediata de destrezas importantes para la adquisición de la lectura por parte del estudiantado como lo son: la motivación, la percepción, la motricidad, la concentración y el lenguaje. También, se realizó una prueba piloto con una maestra y 3 estudiantes con discapacidad auditiva, lo que generó la inquietud de realizar posteriormente un estudio longitudinal para evaluar si la herramienta favorece también al aprendizaje, tanto en estudiantes con síndrome de Down como con discapacidad motriz, visual y auditiva con problemas de lenguaje.
\end{abstract}

Palabras clave: Computación ubicua, lingüística computacional, procesamiento de lenguaje natural, lectoescritura, educación especial, discapacidad.

1. Universidad de Costa Rica. Centro de Investigación en Tecnologías de la Información y la Comunicación (CITIC). Correo electrónico: janio.jadan@ucr.ac.cr

2. Universidad de Costa Rica. Escuela de Orientación y Educación Especial. Correo electrónico: luis.guerrero@ecci.ucr.ac.cr

3. Universidad Tecnológica Indoamérica. Centro de Investigaciones en Mecatrónica y Sistemas Interactivos (MIST).

Correo electrónico: maria.carpiobrenes@ucr.ac.cr

Recepción: 15/1/2016. Aceptación: 16/3/2016 


\begin{abstract}
The development of wireless technologies and mobile devices open the possibility to create new scenarios of application in the field of computational linguistics. One of them is the integration of students with special educational needs in interactive and accessible learning environment. This paper describes a ubiquitous system to strengthen interaction in the process of initial reading of students with Down syndrome. The system is based on the literacy method Picto Phonic (Pifo), which has a tangible user interface with physical objects and 3D letters. These objects can be recognized by Pifo, a puppet bear designed with RFID (Radio Frequency Identification) technology.

At the Special Education Center Centeno Guell in Costa Rica an experimental phase was conducted for evaluating aspects of interaction with activities for the development of phonological awareness in the process of initial reading. In the study participated 4 special education teachers and 6 students with Down syndrome. The results obtained allowed to understand the acquisition of reading skills and the development of cognitive skills: motivation, perception, motor skills, concentration and language. In addition, a pilot test was carried out with a teacher and 3 students with impaired hearing. This event motivated to perform a longitudinal study in order to assess whether the tool also conducive to learning, both in students with Down syndrome as with mobility, visual and hearing disability with language problems.
\end{abstract}

Keywords: Ubiquitous computing, computational linguistics, natural language processing, literacy, special education, disability.

\section{Introducción}

La educación inclusiva es un modelo educativo que busca atender las necesidades de aprendizaje de todos los niños, jóvenes y adultos con especial énfasis en aquellos que viven en situación de vulnerabilidad o discriminación (UNESCO, 2008). El concepto de inclusión o de educación inclusiva suele asociarse a las o los estudiantes con discapacidad o con necesidades educativas especiales; aunque, progresivamente se está adoptando un enfoque más amplio. La exclusión social y educativa son fenómenos crecientes en muchos países del mundo, especialmente en América Latina, que se caracteriza por ser una de las regiones más desiguales del mundo (Andújar et al., 2014).

Costa Rica, un país de 4.7 millones de habitantes, tiene el $10.5 \%$ de su población (493,500 personas) con alguna discapacidad. El $4.4 \%$ de ellos $(21,714)$ tienen edades comprendidas entre 5 y 17 años, de acuerdo con el censo de población del año 2011 (INEC Costa Rica, 2011). En agosto de 2014, se publica el "Primer Informe sobre la situación de la niñez y adolescencia con discapacidad" realizado por el Fondo de las Naciones Unidas para la Infancia (UNICEF) y el Consejo Nacional de Rehabilitación (CNREE), en el cual se indica que la población infantil con discapacidad tiene sus necesidades básicas insatisfechas y se encuentran aún en situaciones de pobreza, marginación y exclusión. Sin embargo, cada vez más se están haciendo esfuerzos para brindar servicios de apoyo en todas las zonas del país, mediante programas fijos e itinerantes. La Ley 8661, Convención sobre los Derechos de las Personas con Discapacidad promulgada por la Organización de las Naciones Unidas (ONU) en 2007 y aprobada en Costa Rica en 2008, es uno de los principales logros, junto con la Ley 7600 , para velar por los derechos de la población con discapacidad (Asamblea Legislativa, 2004; ONU, 2006).

En el ámbito educativo, según el Censo Nacional del 2011 de Costa Rica, existían 23 escuelas de Educación Especial repartidas en las siete provincias del país. Se registraron 124 docentes que trabajan en la enseñanza de estudiantes con discapacidad y se identificaron los porcentajes de niños con discapacidad. La discapacidad 
visual con $35 \%$, problemas intelectuales con $27 \%$, habla con $15 \%$, motriz con $11 \%$, auditiva con $7 \%$ y problemas para utilizar brazos y manos con 5\% (INEC Costa Rica, 2011).

El reto para la Educación Especial no es fácil, ya que se requieren potenciar habilidades que propicien una formación integral de niños y niñas dirigidas hacia su propia vida y al acceso a la vida social, incluyendo las actividades laborales o productivas, con el propósito de que no enfrenten grandes problemas hacia ese fin (Aguilar et al., 2012). En este escenario, se hace necesario el desarrollo de diversas estrategias que contribuyan al fortalecimiento en el proceso de enseñanza-aprendizaje y propicien espacios de interacción e inclusión (Andújar et al., 2014).

Este artículo propone una estrategia desde el área tecnológica, la cual consiste en el desarrollo de una herramienta computacional que apoye el desarrollo de habilidades cognitivas de estudiantes con síndrome de Down. Se enfoca en el proceso de lectura inicial y toma como base el método de lectoescritura Picto Fónico (PiFo) desarrollado por una autora costarricense (Carpio, 2011).

La herramienta está basada en el paradigma de Computación Ubicua, es decir, un sistema que hace invisible la tecnología y se integra al entorno con el fin de que estudiantes con problemas de atención se concentren en las tareas y no tanto en la herramienta tecnológica (Bravo et. al, 2005; Los Santos Aransay, 2009). También, se aplican técnicas de Procesamiento de Lenguaje Natural (PLN, Natural Language Processing) (Cortez, 2009) para fortalecer habilidades de lenguaje. Con la combinación de estas técnicas se busca explorar nuevas formas de interacción que ayuden a los estudiantes a aprender de forma natural y divertida. Se busca, además, observar el tipo de estrategias de enseñanza que puedan desarrollar el profesorado.

La presente investigación es una secuencia de un estudio empírico realizado en España, en la que se introdujo la primera versión del prototipo en un contexto real, también con estudiantes con síndrome de Down (Jadán-Guerrero, Jaen, Carpio, Guerrero, 2015).
En el resto del artículo se aborda la problemática en la sección de "antecedentes". Posteriormente, en la sección de "trabajos relacionados" se resume la investigación llevada a cabo en España. A continuación, se explican los fundamentos del método de lectoescritura PiFo. Luego se presenta el diseño y arquitectura del nuevo prototipo de la herramienta tecnológica. Seguidamente, se expone la fase experimental que se llevó a cabo en el Centro de Educación Especial Fernando Centeno Güell de Costa Rica. En la sección de "resultados y discusión" se reportan los hallazgos de tipo descriptivo, y finalmente se enuncian las conclusiones y trabajo futuro.

\section{Antecedentes}

Algunos estudios realizados en otros países han demostrado que las Tecnologías de la Información y la Comunicación (TIC) están transformando la educación notablemente (Muñoz et al., 2013; Necuzzi, 2013; Vaillant, 2013). También, revelan que, en el área de la Educación Especial, las TIC son altamente motivadoras para los estudiantes y han permitido fomentar la interacción y el desarrollo de habilidades. Reportan que la exposición de la tecnología en el aula de clases agiliza más la enseñanza y aprendizaje, ya que el estudiante tiene interés por investigar, explorar, conocer y descubrir cosas nuevas (Ferreyra, 2009; Wall, 1980).

En países en vías de desarrollo el acceso a la tecnología es limitado. Informes estadísticos del Programa Sociedad de la Información y el Conocimiento (PROSIC) de Costa Rica, reportan que la presencia de tecnología en las aulas aún es escaza. Los recursos tecnológicos en las escuelas continúan siendo restringidos para los docentes. Los pocos que existen son obtenidos de Internet y no todos se adaptan a la necesidad local o idiomática de los docentes (Cordero et al., 2014). Esto abre una oportunidad para desarrollar una propuesta tecnológica que se adapte al contexto costarricense.

En el ámbito educativo, los estudiantes con síndrome de Down generalmente presentan dificultades en algunas áreas de desarrollo de procesos psicológicos básicos como: lenguaje, 
atención, percepción, memoria, pensamiento abstracto y de motivación (Pennington, 2012).

\subsection{Lenguaje}

El lenguaje es una de las áreas de desarrollo con mayores problemas, ya que tienen dificultad para dar respuestas verbales y tienden a dar respuestas motoras, tales como gestos y señas. La comunicación se ve limitada no sólo por sus dificultades para la simbolización y representación, condicionadas por su capacidad intelectual, sino también por aspectos relacionados con la capacidad para emitir y articular palabras, debido a problemas en la respiración y en la motricidad buco facial. Los estudiantes con síndrome de Down presentan mejor capacidad de lenguaje comprensivo que expresivo (Angulo et al., 2015). Revelan que ellos tienen claro lo que quieren decir, pero les cuesta trabajo expresarlo. También, recomiendan que los maestros deben animar a los estudiantes a hablar y no adivinarles el pensamiento y evitar pensar que como no hablan, tampoco entienden.

\subsection{Atención}

La atención es otra área de desarrollo que generalmente presenta dificultades, sobre todo durante períodos prolongados. Con facilidad se distraen frente a la presencia de casi cualquier estímulo (Wisniewski, 1984). Presentan "deficiencias en los sistemas de atención y alerta" que se encuentran muy relacionadas con los problemas para tomar la iniciativa. Así, pueden desconectarse y aislarse si no se provoca una interacción guiada con ellos. Sin embargo, conforme pasa el tiempo pueden adquirir esa capacidad si se utilizan las estrategias adecuadas o experiencias emocionales. Por otro lado, presentan una baja conciencia de sus limitaciones, lo que le provoca dificultades para prever el peligro (Angulo et al., 2015).

\subsection{Percepción}

La percepción visual es una capacidad en la cual los estudiantes con síndrome de Down no presentan muchas dificultades (Yang, 2014).
En general tienen mejor percepción y retención visual que auditiva (Buckley, 2000). Esta característica favorece a su aprendizaje, pues aprenden a desarrollar habilidades a través de la observación. En el contexto educativo un maestro se convierte en un modelo para el estudiante, quien aprende por imitación y repetición, un principio pedagógico fundamental en el proceso de enseñanza-aprendizaje (Vygotski, 2004). El aprendizaje, a partir de la información que se les presente visualmente, se convierte en una oportunidad para introducir el uso de nuevos instrumentos de mediación, como la tecnología (Necuzzi, 2013). Por otro lado, un estudiante con síndrome de Down presenta problemas con la percepción auditiva, ya que no capta bien todos los sonidos y por ello tiene dificultades para responder órdenes (Troncoso, 2009).

\subsection{Memoria}

La memoria se divide en corto plazo y largo plazo, pero también existe la memoria sensorial, relacionada con los sentidos (Sternberg, 1999). Los estudiantes con síndrome de Down presentan dificultades con la memoria a corto y largo plazo y es mejor su memoria visual que la auditiva, como se explicó anteriormente. Les resulta difícil realizar los procesos de conceptualización, generalización, transferencia de aprendizajes y abstracción. Necesitan más tiempo para aprender y responder a ciertos estímulos. Además, les es complicado entender y seguir varias instrucciones a la vez y les es difícil generalizar lo que aprenden (Escamilla, 1983). Es por esta razón que los maestros requieren de actividades repetitivas, las cuales muchas veces se tornan tediosas y aburridas (Angulo et al., 2015).

\subsection{Inteligencia}

Los estudiantes con síndrome de Down desarrollan la inteligencia a lo largo de su vida, si se les ofrecen oportunidades de aprender, mediante la interacción permanente con la familia, escuela y sociedad (Angulo et al., 2015). Algunas investigaciones ponen en evidencia que si se utilizan estrategias adecuadas los 
estudiantes son capaces de desarrollar muchas habilidades, entre ellas la lectura y la escritura (Buckley, 2000; Epstein, et al., 2002; O’Malley et al., 2004). Otros estudios también indican que el desarrollo de la inteligencia de los estudiantes con síndrome de Down dependerá en forma extrema de la calidad de educación y ambiente en el que la persona crezca y se desarrolle. El fomento de las habilidades sociales e inteligencia emocional han hecho incrementar considerablemente su capacidad adaptativa (Ramos, 2004). Es, por tanto, muy conveniente que los maestros desarrollen la inteligencia en todos sus ámbitos $\mathrm{y}$ vertientes.

\subsection{Motivación}

La motivación es otra dificultad que presenta el estudiantado con síndrome de Down, pues se ve limitada cuando se tiene que enfrentar a tareas más difíciles y menos agradables (Rodríguez, 2004). A veces muestran episodios de resistencia creciente al esfuerzo en la realización de una tarea debido a la poca motivación y falta de interés, y no tanto por lo que conocen (Wall, 1980). El ambiente emocional en que el estudiante se desarrolle es determinante para cambiar su actitud y forma de manifestar sus sentimientos. Un ambiente calmado, tranquilo, comunicativo y cordial creará unas condiciones para un adecuado desarrollo emocional. Un ambiente tenso o frío fomentará la inquietud, frustración e inseguridad del estudiante (Angulo et al., 2015; Rodríguez, 2004).

De forma general, se han descrito las principales dificultades que presenta el estudiantado con síndrome de Down, las cuales pueden incidir en el aprendizaje de la lectura y la escritura. Sin embargo, con una adecuada intervención educativa, que incluya estrategias y recursos que generen espacios de interacción y manipulación, se pueden estimular satisfactoriamente estas, como se muestran a continuación.

\section{Trabajos relacionados}

La investigación que se presenta en este artículo está basada en dos estudios preliminares realizados en España. El primero hace referencia al diseño de un repositorio web de objetos de aprendizaje denominado TIC@ULA(Tecnologías de la Información y la Comunicación en el aula) (Jadán-Guerrero, López, y Guerrero, 2014). La estructura del repositorio se basó en el estándar de metadatos de objetos de aprendizaje (LOM, Learning Object Metadata) propuesto por el Instituto de Ingeniería Eléctrica y Electrónica (IEEE, Institute of Electrical and Electronics Engineers). Dado que los metadatos de los objetos de aprendizaje proporcionan una información adicional a materiales digitales que facilitan su clasificación y recuperación (Barker, 2005); se estructuró la información de un método de lectoescritura global para estudiantes con síndrome de Down, propuesto por dos autoras españolas (Troncoso, 2009). Además, se diseñó un prototipo de un kit de objetos tangibles basado en computación ubicua, con el fin de que un maestro pueda diseñar estrategias de aprendizaje para desarrollar habilidades de lenguaje, atención, percepción, memoria, pensamiento abstracto y de motivación. La plataforma en conjunto fue evaluada por 5 expertos en Educación Especial a través de la técnica pensando en voz alta (TAP, thinking aloud protocols.), quienes también demostraron su interés y potencial uso.

El segundo estudio hace referencia a una investigación experimental llevada a cabo en ASINDOWN, una institución de Educación Especial en Valencia, España. Para ello, se adaptó la plataforma con el método de lectoescritura global y se diseñó un segundo prototipo denominado KITERACY (Kit for Literacy) (Jadán-Guerrero, Jaen, et al., 2015). El kit usa un sistema de reconocimiento de objetos del entorno por medio de un sensor que usa tecnología RFID (Identificador de Radio Frecuencia). Esta tecnología permite identificación mediante radiofrecuencia sin necesidad de contacto directo entre un microchip (RFID tag) y un dispositivo lector (Kriara et al., 2013). Se diseñaron tres tipos de instrumentos de mediación: tarjetas de cartulina, tabletas con información digital multimedia de Tic@ula y 24 objetos tangibles agrupados en 4 categorías (animales, frutas, hogar, entretenimiento). 
En el estudio experimental participaron 4 maestras de Educación Especial, así como 10 niños y 2 niñas con síndrome de Down con edades comprendidas entre los 4 y los 8 años. El estudio fue descriptivo y el objetivo fue explorar el tipo de interacción generada al utilizar la tecnología como instrumento de mediación de enseñanza de la lectura. Para las observaciones se consideraron dos escenarios: aprendizaje mediado con un maestro y aprendizaje autónomo. La metodología consistió en una encuesta inicial a los docentes para determinar el nivel de conocimiento de las tecnologías y registrar los datos de los niños y niñas participantes. Posteriormente, con un documento de autorización de los padres de familia se programaron sesiones de 10 minutos por cada escenario de aprendizaje (maestroestudiante y aprendizaje autónomo) y por cada instrumento de mediación (tarjetas, digital y tangible); es decir, 6 sesiones en total por cada niño. Este proceso se llevó a cabo durante un mes, y al final se aplicó una encuesta a los docentes con el fin de conocer su experiencia y retroalimentación. Los resultados revelaron que estas herramientas propiciaron espacios de interacción lúdicos y generaron emociones positivas en maestros, niños y niñas. Además, se evidenció que el uso de las interfaces tangibles puede favorecer a la conexión del pensamiento concreto con el abstracto.

El tercer estudio hace referencia a los problemas técnicos que se detectaron en la evaluación experimental. Problemas relacionados con la accesibilidad de la interfaz tangible, debido a las dificultades de motricidad que presentan los estudiantes con síndrome de Down. También, en el uso de algunos materiales que cubren las etiquetas RFID incrustadas o pegadas en los objetos. (Jadán-Guerrero, Guerrero, López, Cáliz, \& Bravo, 2015)technology can support specific strategies that will help children learn to read. This paper introduces a Tangible User Interface (TUI. En el artículo se reportaron los resultados de las mediciones realizadas con dos sensores RFID: Phidget RFID y Tertium Icekey HF RFID. Para cada sensor se utilizaron tres tipos de etiquetas pasivas RFID y se realizaron mediciones por cada caso, usando 8 materiales: aluminio, vidrio, metal, cartón, plástico, porcelana, caucho y madera. Los materiales que mayor dificultad presentaron en el reconocimiento fueron: metal, aluminio y caucho, ya que los metales inhiben la onda de propagación de radiofrecuencia y el caucho disminuía la señal. Debido a esto, los estudiantes tenían dificultades en ciertos objetos, como los animales de caucho; sin embargo, aprendieron a girarlos hasta que sea reconocido. Se determinó que la distancia promedio necesaria para que los objetos con las etiquetas incrustados sean reconocidos fue de $7.5 \mathrm{~cm}$. Finalmente, se encontraron también dificultades cuando la posición de las etiquetas estaba en orientación perpendicular al sensor.

Estas experiencias sirvieron para diseñar un tercer prototipo con las mejoras respectivas y probar la escalabilidad de la plataforma con un nuevo método de lectoescritura: el método Picto Fónico (PiFo).

\section{Método de Lectoescritura PiFo}

El método Picto Fónico (PiFo) para la enseñanza de la lectoescritura fue propuesto por la profesora costarricense María de los Ángeles Carpio Brenes. Este pertenece a la categoría de los métodos fonéticos y acuña un concepto de Estrategias Picto Fónicas (Carpio, 2011). Su nombre se debe a la integración de dos aspectos, el pictograma, basado en un dibujo que tiene o se asocia a la forma de la letra en estudio; y el fónico, porque el dibujo ilustra una palabra clave cuyo sonido inicial es el correspondiente a la letra representada. El estudiante aprende a reconocer los grafemas y a asociarlos con su fonema mediante el sonido inicial de la palabra clave y el dibujo que ilustra esta, estimulando así un proceso dual de aprendizaje de la lectura. En la Figura 1 se muestran algunos recursos del método.

El método propone una secuencia didáctica que guía a cada maestro en la enseñanza de la lectura y la escritura mediante el uso de recursos dinámicos como canciones, videos, fichas y folletos que se encentran en los discos compactos. También, se muestran las tarjetas con los 


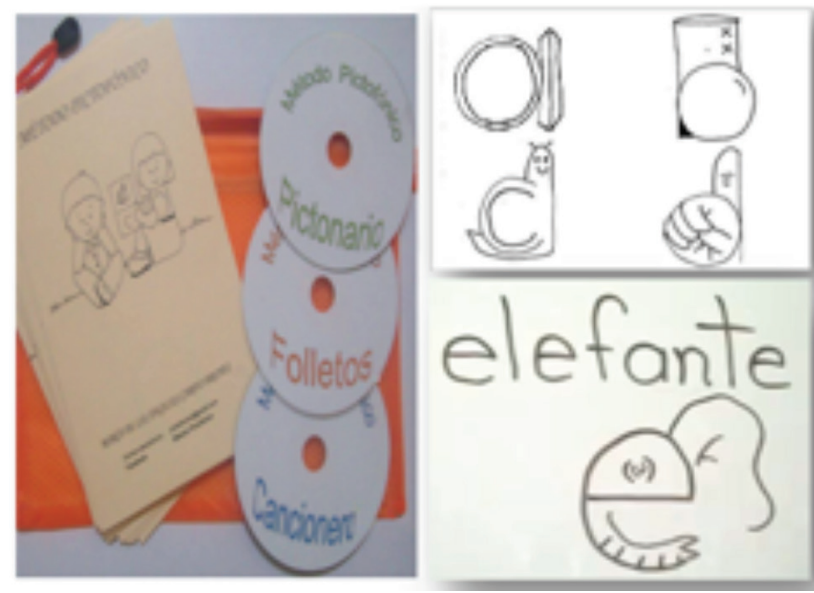

FIGURA 1

Método PiFo; 31 Tarjetas y discos compactos con canciones y videos.

grafemas de las letras a, b, c, d y e. En cada caso se asocia un fonema con las siguientes palabras: /a/ con anillo, /b/ con bota, /k/ con caracol, /d/ con dedo y /e/ con elefante.

Las Estrategias Picto Fónicas que aplican el método PiFo estimulan un proceso sintético de aprendizaje de la lectoescritura en cuatro etapas: perceptual, decodificación, comprensión y redacción. En el Cuadro 1 se resumen estas etapas, con los subprocesos y el tiempo en semanas sugeridas para el desarrollo del sistema ortográfico y las representaciones fonológicas.

\section{CUADRO 1}

Etapas del método PiFo

\begin{tabular}{llll}
\hline No. & Etapa & Procesos & \\
\hline 1 & Perceptual & Lectura fotográfica \\
& & $\begin{array}{l}\text { Conciencia fonológica. } \\
\text { Vocales } \\
\text { Conciencia fonémica } \\
\text { Fusión silábica }\end{array}$ & \\
2 & Decodificación & Reconocimiento de palabras & \\
& & Comprensión lectora & $6-35$ \\
& Estructurar oraciones & \\
3 & Comprensión & & $8-35$ \\
4 & Redacción & & $10-35$ \\
\hline
\end{tabular}

Fuente: (Carpio, 2011)

En la primera etapa se introducen las destrezas motrices gruesas y finas, conceptuales, lingüísticas y sociales, mediante actividades que permiten preparar a los estudiantes en el proceso de lectura inicial. Se estimula la lectura logográfica, por medio de carteles del ambiente: DAMAS, CABALLEROS, ABIERTO, CERRADO, ENTRADA, SALIDA, entre otros. La conciencia fonológica es indispensable para el proceso alfabético y se hace con la presentación 
de las vocales para el proceso de correspondencia grafema-fonema, con la asociación del pictograma y la letra, y con el sonido inicial de la palabra clave con el fonema. En esta fase, se enfatiza la enseñanza del trazo con dibujos, los cuales modelan los y las docentes a partir de los videos que incluyen el método. Es indispensable el estímulo auditivo con las canciones que sugiere el método.

En la etapa de decodificación, se proponen tres fases: las reglas de correspondencia grafema-fonema (RCGF) para la introducción de las letras consonánticas; el ensamblaje o fusión silábica de las consonantes con cada vocal; y la lectura de palabras para desarrollar el reconocimiento automático de estas. Se trabaja simultáneamente la escritura, caligrafía y ortografía.

En la etapa de comprensión, se estimula desde la primera serie silábica con oraciones sencillas como "Memo ama a Ema", "Mi mamá me mima". Para el desarrollo del procesamiento semántico, se acompañan las oraciones con ilustraciones que estimulan la construcción de representaciones mentales del contenido.

La etapa de redacción, se introduce cuando los y las estudiantes tienen suficiente vocabulario como para estructurar oraciones en el esquema de sujeto-verbo-predicado, mediante tres dibujos clave: una carita (sujeto), un signo de pregunta (verbo) y una nube (predicado). El método provee, en un $\mathrm{CD}$, todo el material gráfico que requiere la o el maestro para el trabajo directo con el grupo de estudiantes o para su atención individual.

De acuerdo con la autora del método y con fines de evaluación, se aplicó el componente tecnológico en la primera etapa, enfocado principalmente en las vocales y conciencia fonológica. Para ello, se realizó una modificación del sistema computacional Kiteracy, que se describe a continuación.

\section{Kitercy PiFo}

Kiteracy PiFo incorpora una interfaz basada en computación ubicua y técnicas de procesamiento de lenguaje natural. Este prototipo se enfoca en la etapa perceptual del método
PiFo, orientada al desarrollo de la conciencia fonológica y el establecimiento de las reglas de correspondencia grafema-fonema. Este método se apoya mediante tarjetas de cartulina y tarjetas digitales con pictogramas que representan la palabra clave de cada vocal. Incluye, además, las vocales en 3D, adheridas a una etiqueta RFID tipo tarjeta, y utiliza también un objeto físico, un juguete de plástico o madera que representan la palabra clave de cada vocal (por ejemplo, el grafema $<\mathrm{i}>$ se asocia con una iglesia), a la cual se le incrusta una etiqueta RFID. Dado que este nuevo prototipo pretende hacer invisible la tecnología y generar niveles de interacción y emoción, se usa a PiFo, un oso perezoso de peluche, el cual tiene incorporado un sensor RFID. El peluche esconde en su libro el sensor RFID y actúa de asistente de lectura. En la Figura 2 se muestra el prototipo con los objetos y el sensor.

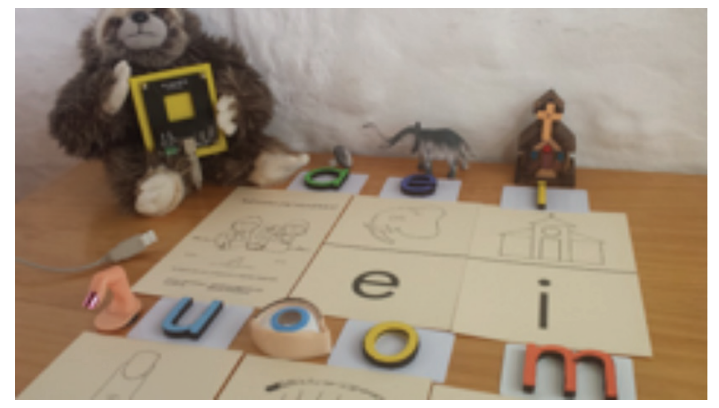

FIGURA 2

Kitaracy PiFo con tarjetas de cartulina, vocales 3D y objetos tangibles.

El peluche se conecta a cualquier computador por medio de un cable USB. El prototipo incluye un software que gestiona las configuraciones del sistema y procesa la información que recoge el sensor. La etiqueta incorporada en cada objeto o letra tiene un código único, similar al código de barras o códigos $\mathrm{QR}$, con la diferencia de que la tecnología RFID no requiere precisión al momento de acercar una marca o patrón en el sensor, sino que funciona por aproximación (entre 0 a $12 \mathrm{~cm}$ ). El código es recogido por el sensor y enviado al software del computador. El software, a través de internet, consume un servicio web de 
la plataforma Tic@ula y gestiona la presentación de los recursos a través de un algoritmo de recuperación de información.

El algoritmo de recuperación de información busca el código en la estructura IEEELOM del repositorio web, extrae las tarjetas digitales con los pictogramas, grafemas y videos que provee el método. Esta información es enviada a cualquier computador, tableta o teléfono celular que haya iniciado una sesión de usuario. Previamente, la maestra debe ejecutar el software en un computador o una aplicación en uno o varios dispositivos móviles con la información de inicio de sesión como: número, nombre de la maestra, nombre del estudiante, tiempo para reproducir el sonido, interfaz (digital o tangible), idioma, voz y los grafemas por estudiar, como se muestra en la Figura 3.

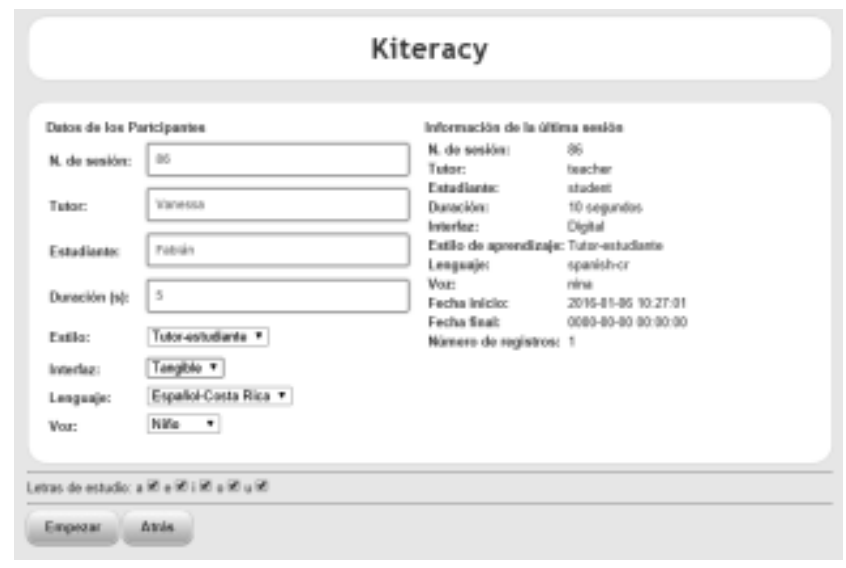

FIGURA 3

Inicio de sesión en el software Kiteracy PiFo.

El número de sesión es el que permite que varios dispositivos reciban la información que lee el sensor. Esto quiere decir, que pueden haber sesiones simultaneas en el caso que varias maestras se conecten al sistema. A continuación, se detallan algunas características del sistem en aspectos relacionados con Computación Ubicua y Procesamiento de Lenguaje Natural.

\subsection{Computación Ubicua}

La Computación Ubicua fue descrita por primera vez por Mark Weiser en 1991. La esencia de su visión era la creación de dispositivos computacionales con capacidad de comunicación que pudieran integrarse de forma inapreciable en la vida cotidiana de las personas. Lo anterior, con el fin de permitirles que se centren en las tareas que deben hacer y no en las herramientas que deben usar (Los Santos Aransay, 2009; Weiser, 1991).

Kiteracy PiFo pone en práctica este paradigma para apoyar el proceso de aprendizaje de forma natural e intuitiva, no solo por la ubicuidad, sino por su interfaz tangible, dado que brinda mayor accesibilidad (Ishii et al., 1997). Esto quiere decir que no basta con que sea fácil de usar, sino también que cualquier persona pueda usarlo, independientemente de su diversidad funcional, capacidad sensorial, cognitiva o motriz. Con este nuevo prototipo, se espera que los estudiantes con síndrome de Down desarrollen habilidades cognitivas, por ejemplo, la ubicuidad favorece a que los estudiantes se concentren en las letras y palabras antes que en las tabletas a través del uso de la metáfora del "oso lector" para favorecer la interacción y motivación. La 
tecnología RFID favorece a los estudiantes con dificultades motrices para que puedan acercar los objetos al sensor sin mayor precisión. La interfaz tangible favorece la percepción visual y táctil, así como el pensamiento abstracto a partir del concreto. Por último, favorece el uso de procesamiento de lenguaje natural que ayuda a desarrollar el lenguaje y comunicación.

\subsubsection{El Procesamiento de Lenguaje Natural}

El Procesamiento de Lenguaje Natural (PLN, Natural Language Processing) es una disciplina de la Inteligencia Artificial, la cual se ocupa de la formulación e investigación de mecanismos computacionalmente eficaces para la comunicación entre personas o entre persona y máquinas, por medio de lenguajes naturales (Cortez et al., 2009).

Kiteracy PiFo aplica técnicas de procesamiento de lenguaje natural para combinar la información gráfica y tangible con la generación de fonemas y pronunciación de sílabas y palabras. El sistema tiene una estructura escalable que le permite incorporar idiomas o dialectos con diferentes voces (hombre, mujer, niño y niña). Para ello, se aplican archivos de audio en formato MP3 previamente grabadas. Actualmente, el sistema provee la voz de la autora del método PiFo y las voces de un niño y una niña costarricenses. El uso de audios pregrabados en lugar de librerías Text-to-Speech, se debe a que es necesario reproducir fonemas y no sólo la pronunciación de la letra, por ejemplo, para la letra "m" sonaría "eme" y no el fonema $/ \mathrm{m} /$ que suena "mmm". Otra razón por la que se usaron archivos de audio MP3, se debe a que se necesitaba enfatizar en la pronunciación de las palabras, de acuerdo con la teoría que propone el método para desarrollar la conciencia fonología.

El sistema actualmente identifica una letra y reproduce el fonema correspondiente, pero si se pasan por el sensor una consonante y una vocal juntas, lee la sílaba. El algoritmo en este caso utiliza librerías Text-to-Speech para el reconocimiento de sílabas y palabras formadas por varias letras tangibles, como el ejemplo que se muestra en la Figura 4.

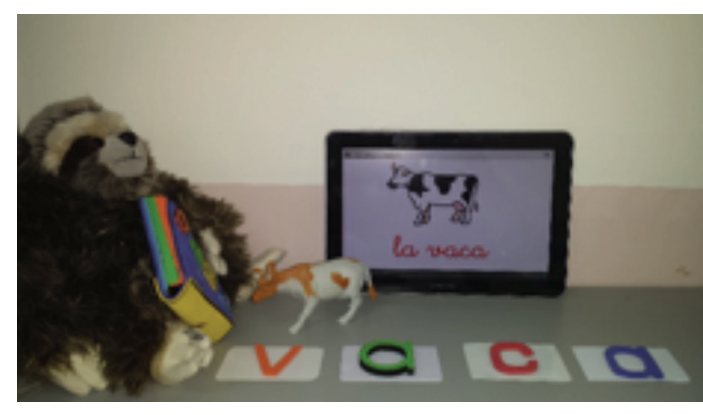

FIGURA 4

Asistente de lectura PiFo que identifica letras (fonemas), sílabas y palabras.

El algoritmo identifica el código de cada objeto y el tiempo entre cada lectura. En el ejemplo, identifica el código del juguete y pronuncia "vaca", si solo se acerca la letra tangible de la "v" genera el fonema /b/. Si se deslizan las dos letras junta por el sensor pronuncia "va". El algoritmo registra el tiempo de lectura entre cada letra para determinar si están juntas y no sean tratadas individualmente. En el caso de palabras completas, el algoritmo determina el tiempo de lectura entre las letras para formar la palabra y reproducirla con las librerías Text-to-Speech. El objetivo de esta funcionalidad fue la de convertir a Kiteracy PiFo en un asistente de lectura, logre motivar el aprendizaje autónomo de los estudiantes o bien apoyar a los padres de familia en el hogar con trabajo de refuerzo.

Para evaluar el funcionamiento de la herramienta en un contexto real, se hizo acercamientos con uno de los centros de Educación Especial más importantes de Costa Rica. A continuación, se detalla el estudio experimental.

\section{Estudio Experimental}

Por medio de la autora del método PiFo se pudo contactar a la Directora del Centro de Educación Especial Fernando Centeno Güell de Costa Rica, quien mostró interés en el proyecto de investigación y abrió las puertas de dicho Centro.

Primero, se realizó un grupo focal con 15 maestras de Educación Especial, en el cual 
se expuso la propuesta computacional y se recabó información para ajustar el prototipo. Posteriormente, en coordinación con la directora y el equipo de investigación, se diseñaron las tareas para evaluar aspectos de interacción y desarrollo de conciencia fonológica en el proceso de lectura inicial. Finalmente, se realizó un documento de consentimiento informado y en coordinación con la directora del Departamento de Discapacidad Intelectual, se socializó con los padres de familia de estudiantes con síndrome de Down.

\subsection{Participantes}

En el estudio participaron 4 maestras de Educación Especial especializadas en retardo mental y 6 estudiantes con síndrome de Down (tres niñas). La edad cronológica promedio de los estudiantes era de 8.33 años con una desviación estándar de 1.63. Todos los estudiantes estaban en el nivel de competencia curricular correspondiente a 4-5 años y estaban en el proceso de lectura inicial. Según los datos demográficos proporcionados por las maestras, en el Cuadro 2 se resume el nivel de desarrollo de las funciones cognitivas.

En el Cuadro 2 se listan las funciones cognitivas que se detallaron en una encuesta demográfica realizada a las maestras. En cada uno de los casilleros de la escala se registra el número de estudiantes y, como se puede observar, la mayoría se concentra en un nivel regular y bueno en casi todas las funciones cognitivas, exceptuando unos pocos con nivel muy bueno.

CUADRO 2

Nivel de desarrollo de funciones cognitivas $\mathrm{M}=$ Mala, $\mathrm{R}=$ Regular, $\mathrm{B}=\mathrm{Buena}, \mathrm{MB}=$ Muy Buena, $\mathrm{E}=$ Excelente

\begin{tabular}{|c|c|c|c|c|c|}
\hline Función cognitiva & M & $\mathrm{R}$ & B & MB & $\mathrm{E}$ \\
\hline Percepción visual & & 4 & 2 & & \\
\hline Percepción auditiva & & 2 & 3 & 1 & \\
\hline Emitir y articular palabras & & 5 & 1 & & \\
\hline Simbolización y representación & & 3 & 3 & & \\
\hline Atención & & 2 & 3 & 1 & \\
\hline Discriminación & & 3 & 2 & 1 & \\
\hline Selección & & 3 & 3 & & \\
\hline Denominación & & 2 & 3 & 1 & \\
\hline Memoria a corto plazo & & 2 & 3 & 1 & \\
\hline Memoria a largo plazo & & 2 & 3 & 1 & \\
\hline Coordinación visomotora (iniciativa) & & 2 & 4 & & \\
\hline Coordinación visomotora (control) & & 3 & 2 & 1 & \\
\hline
\end{tabular}

Fuente: Resumen de la encuestra realizada a las 4 maestras.

\subsection{Instrumento de experimentación}

Los instrumentos de experimentación fueron de tres tipos: 5 tarjetas de cartulina que provee el método, cada una tiene una vocal y un grafema de la vocal. También, se usó 25 tarjetas pequeñas (flash cards), 5 por cada vocal, cada una con un pictograma y la vocal. Se usó una de 10 pulgadas con información digital multimedia de las mismas 30 tarjetas de cartulina. La tableta tenía conexión a internet para acceder al repositorio web 
Tic@ula. Finalmente, 5 vocales tangibles y 5 objetos tangibles (uno por cada vocal).

\subsection{Procedimiento}

Se comenzó con una encuesta dirigida a las maestras, con el fin de recabar información demográfica de los estudiantes e indagar el grado de acercamiento de las maestras a la tecnología; así como, para conocer las estrategias y recursos que usan para enseñar a leer a los estudiantes con síndrome de Down. Posteriormente, se realizó la fase experimental que se describe en el siguiente párrafo. Al finalizar la fase experimental, se realizó una encuesta "post-test" para recabar el criterio de las maestras, dada su experiencia y su apreciación en la interacción de los estudiantes, con relación al proceso tradicional.

El procedimiento de la fase experimental fue similar a la que se llevó a cabo en España. Es decir, se consideraron dos escenarios: aprendizaje mediado con un maestro y aprendizaje autónomo. En cada escenario se programaron sesiones de 10 minutos por cada interfaz (tarjetas, digital y tangible), en total 6 sesiones por cada estudiante. Las tareas en el escenario maestra-estudiante pueden resumirse en seis pasos:

1. La maestra selecciona una vocal (cartulina, digital o tangible) y muestra al estudiante.

2. La maestra verbaliza el fonema mientras el estudiante mira el grafema.

3. La maestra pide al estudiante que repita el fonema.

4. La maestra asocia el grafema con el pictograma (en cartulina señala con el dedo cómo se forma el grafema, en digital muestra un vídeo, en tangible selecciona un objeto).

5. La maestra motiva al estudiante a que repita el fonema y el nombre del grafema.

6. La maestra desarrolla algunas estrategias de aprendizaje (selección, memoria, discriminación).

Para el caso de aprendizaje autónomo, las actividades se resumen en cuatro pasos:
1. La maestra entrega al estudiante los recursos educativos (30 tarjetas, tableta o 10 objetos tangibles).

2. La maestra le recuerda al estudiante cómo las usaron en la sesión maestra-estudiante.

3. La maestra pide al estudiante que "juegue" libremente durante 10 minutos.

Todas las sesiones fueron grabadas en vídeo. Además, participaron dos observadores que anotaban algunos eventos importantes.

\section{Resultados y Discusión}

Los resultados que se describen en este artículo son de tipo descriptivo y se han estructurado en cuatro secciones: interfaz con tarjetas, interfaz digital, interfaz tangible y los resultados de la encuesta post-test.

\subsection{Interfaz con tarjetas}

En el escenario maestra-estudiante, las cuatro maestras comenzaron seleccionando la tarjeta de la vocal "a" y continuaron en orden hasta la "u". Mostraron cada tarjeta al estudiante, verbalizaron el fonema de la vocal y pidieron al estudiante que repitiera. Se observó que las maestras fueron las que manipulaban las tarjetas, los estudiantes solo miraban sin tocarlas. Solo una maestra tomó la mano del estudiante para seguir el trazado del grafema con su dedo. Las tarjetas permitieron que las maestras generaran estrategias de selección, por ejemplo, ubicaban algunas tarjetas sobre la mesa y preguntaban al estudiante dónde está determinada letra o pictograma. En este caso, algunos estudiantes solamente señalaban la tarjeta sin verbalizar, la maestra tenía que motivar para que verbalizaran. Una estrategia similar fue la de adivinanza, en la cual daban las características del pictograma y el estudiante debía seleccionar y verbalizar. En algunos pictogramas, los estudiantes mostraron interés, pero se notó que pasados los cinco minutos expresaban aburrimiento, se distraían o se recostaban sobre la mesa como señal de cansancio.

Una de las maestras indicó que antes había trabajado con el método PiFo con estudiantes de 
preescolar y que la enseñanza de las vocales fue muy rápida; en una semana ya podían identificar todas las vocales. Sin embargo, en estudiantes con problemas cognitivos, le ha tomado más tiempo, ya que de una semana a otra se olvidaban y era necesario retomar, dependiendo de cada caso puede durar hasta un mes solo en las vocales.

En el escenario de aprendizaje autónomo, tres estudiantes manipularon las tarjetas de forma similar a las maestras, es decir, primero las agruparon y luego fueron seleccionando un tras una. En cada tarjeta se detenían unos pocos segundos, miraban los pictogramas, pero no verbalizaban, excepto por unas pocas que les llamaba la atención. Dos estudiantes no mostraron mayor interés y no lograron permanecer los 10 minutos en la actividad, las dejaban en la mesa y se distraían en algún otro evento. Un estudiante ni siquiera quiso tocar las tarjetas y permaneció sentado.

\subsection{Interfaz digital}

En el escenario maestra-estudiante, se observó un fenómeno interesante, a los seis estudiantes les llamó la atención la presencia de la tableta, cuatro de ellos cambiaron de posición, se sentaron más erguidos. Incluso dos de ellos quisieron tomar la iniciativa, tocando la pantalla antes de que la maestra les diera las instrucciones. Se sorprendían al escuchar que la tableta verbalizaba la vocal o pictograma que seleccionaban. Les llamó la atención el vídeo de trazo del grafema. Dos maestras manipularon la tableta igual que las tarjetas de cartulina, es decir, ellas eran las que sostenían la tableta y seleccionaban los pictogramas, sin que su estudiante interactúe con la tableta. Los estudiantes, en estos casos, se limitaron a mirar y verbalizar. Las otras dos maestras sí permitieron que sus estudiantes tocaran el pictograma solicitado o incluso los tomaban de la mano y con el dedo señalaban juntos un determinado pictograma. Un problema que se presentó con la interfaz digital fue que los estudiantes, con el propósito de experimentar, tocaban otros botones y se salían de la aplicación.
En el escenario de aprendizaje autónomo, los seis estudiantes se limitaron a seleccionar los pictogramas, los miraban por pocos segundos sin verbalizar, excepto en pictogramas de animales y frutas que les llamaba la atención y verbalizaban en voz baja o levantaban la cabeza para mirar a su profesora y verbalizaban. Parece que esta interfaz causó mayor interés a los estudiantes, algunos de ellos no querían dejarla al finalizar los 10 minutos de la sesión. Sin embargo, se observó que limitó la generación de estrategias a las maestras. Primero, porque no tenían la flexibilidad para manipular las tareas como lo hacen con las de cartulina; segundo, porque la pantalla de 10 pulgadas resultó muy pequeña para que pudieran interactuar la maestra y el estudiante. Sólo dos maestras intentaron generar una actividad de selección al decir las características de un concepto y pedir al estudiante que lo buscara.

\subsection{Interfaz tangible}

En el escenario maestra-estudiante, se evidenció interés tanto por las maestras como por los estudiantes. En la Figura 5, se muestra la expresión de la maestra al decir: “Está chivísima!”.

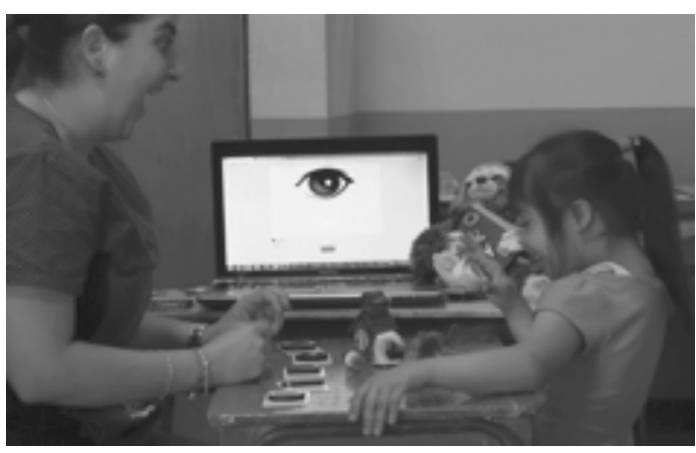

FIGURA 5

Sesión con la interfaz tangible.

La emoción de los seis estudiantes fue evidente, se sorprendieron que el "oso perezoso pudiera leer", les emocionó escuchar la verbalización del sistema con la voz de niña o niño que previamente seleccionó la maestra. Se observó 
una mayor motivación de los estudiantes con relación a las otras interfaces, según las frecuencias de emociones positivas contabilizadas en el análisis de los vídeos. En el período de 10 minutos se tuvo un promedio de 2,5 en la interfaz de papel, 3 en la interfaz digital y 4 en la interfaz tangible. Fue notoria su iniciativa para verbalizar sin necesidad que las maestras los motivara, lo hacían después de escuchar la verbalización del sistema. A pesar de que se usa la computadora para mostrar en pantalla los pictogramas y grafemas, los estudiantes no la tocaban y se concentraban en los objetos de estudio. Tres maestras dejaron que los estudiantes tomaran la iniciativa y únicamente intervinieron para ayudar a ubicar el objeto cerca del sensor o para dar retroalimentación sobre el objeto de estudio.

En el escenario de aprendizaje autónomo, los seis estudiantes sin la presencia de la maestra verbalizaron los fonemas y palabras después de escuchar el sistema. También, se evidenció la accesibilidad del sistema, los estudiantes aprendieron a usarla rápidamente e identificaron cuando el sistema ya reconocía el objeto al escuchar un "beep" del sensor. Se vieron motivados durante toda la sesión, pasaban una y otra vez los objetos y en su mayoría verbalizaban, reían y hacían gestos de emoción.

\subsection{Encuestas post-test}

En las encuestas post-test, las seis maestras concuerdan que las herramientas tecnológicas favorecen la motivación de los estudiantes, pues les permiten generar nuevas estrategias de enseñanza. Además, proveen un espacio de interacción y de negociación, fomentan la atención y coordinación de los estudiantes, generan canales de comunicación que promueven y median la interacción social entre la maestra y el estudiante y permiten reforzar el desarrollo del lenguaje, logran niveles mayores y significativos de atención. Los objetos abstractos, que se presentan en una lámina o pantalla de algún dispositivo, pueden ser representados de forma física. De esta manera, perciben la globalidad de las cosas con objetos físicos de su entorno para lograr una transición lenta del aprendizaje abstracto.
Es decir, el objeto constituye la parte concreta, el vídeo del grafema la parte semi-concreta y el pictograma sería lo abstracto.

También, sugirieron ampliar otras actividades, por ejemplo, una de las maestras dijo que el sistema está "divino" y que le encanta. Por tanto, resaltó que se podría crear una estrategia de "caja de sorpresas" para ir sacando los objetos y explorar con el estudiante. Otra maestra sugirió la narración de cuentos, en la cual aparezca un escenario en la pantalla del computador. Cada vez que se pase un objeto por el sensor podría aparecer dentro del escenario y con ellos crear una historia para motivar al estudiante a que interactúe, verbalice y participe en el cuento.

\section{Conclusiones}

Con los resultados de la fase experimental y de las opiniones de las maestras, parece ser que Kiteracy PiFo generó un mayor grado de interacción que la interfaz digital y la interfaz de las tarjetas. La interfaz tangible propició espacios de interacción lúdicos y generaron emociones positivas en los maestros y estudiantes. El uso de los sistemas ubicuos parece ser la nueva tendencia en el futuro para la Educación Especial, ya que favorece la accesibilidad y participación en ambientes inclusivos. El procesamiento de lenguaje natural parece fomentar la comunicación y verbalización de los estudiantes. La verbalización se hace natural, incluso sin la presencia de la maestra, lo cual puede favorecer el aprendizaje autónomo. Los resultados obtenidos también permitieron evidenciar que los estudiantes mostraron mayor concentración, percepción visual y auditiva, motricidad al manipular objetos, atención y concentración al enfocarse en los objetos y no en la tecnología, memoria y abstracción al recordar el procedimiento para hacer sonar cada letra y, finalmente, seguir el lenguaje gracias a un modelo de verbalización.

\section{Trabajo futuro}

Esta experiencia ha permitido identificar otras necesidades latentes, como las que se pueden listar a continuación: 
1. Evaluación con interfaces híbridas (combinar los recursos de las tres interfaces).

2. Interfaz tangible multisensor para mejorar la precisión.

3. Interfaz offline para no depender de Internet.

4. Asistente de lectura para identificar letras, sílabas y palabras.

5. Estudio grupal para la inclusión educativa.

6. Evaluación con otras discapacidades.

7. Estudio longitudinal para evaluar el aprendizaje de la lectura.

Con respecto a las dos últimas, se realizó una prueba piloto con una maestra de audición y lenguaje y tres estudiantes, dos niñas y un niño con discapacidad auditiva y de lenguaje. En la figura 6 , se muestra la sesión de trabajo con la interfaz tangible.

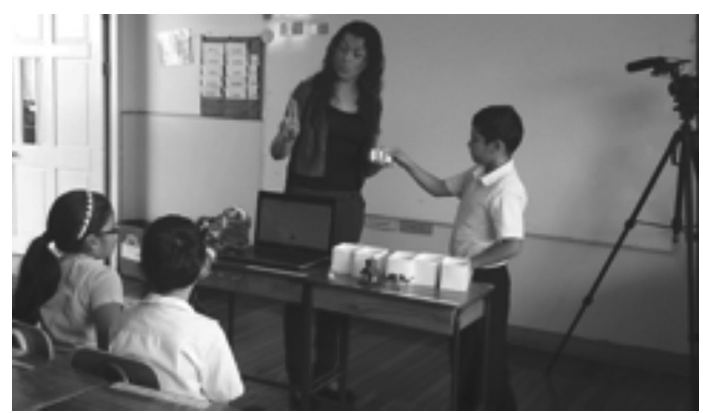

FIGURA 6

Sesión con estudiantes con discapacidad auditiva $\mathrm{y}$ de lenguaje.

Los resultados encontrados, motivaron a las autoridades del Centro de Educación Especial a llevar un estudio longitudinal que permita hacer una evaluación del desempeño en el aprendizaje de la lectura, tanto para estudiantes con síndrome de Down, como para estudiantes con discapacidad motriz, visual, auditiva y lenguaje.

Es importante indicar que está en proceso la abstracción de variables que permitan evaluar cuantitativamente y mediante un análisis estadístico, determinar diferencias significativas en el uso de las tres interfaces.

\section{Agradecimientos}

Un agradecimiento especial a todos los niños y niñas del Centro de Educación Especial Fernando Centeno Güell, así como a las autoridades y maestras por la apertura y participación en el desarrollo de esta investigación. Un reconocimiento al Centro de Investigación en Tecnologías de la Información y la Comunicación de la Universidad de Costa Rica (CITIC), a la Secretaría Nacional de Educación Superior, Ciencia y Tecnología del Ecuador (SENESCYT), a la Universidad Tecnológica Indoamérica de Ecuador (UTI), al Sistema de Estudios de Posgrado de la Universidad de Costa Rica (SEP) por su apoyo logístico y financiero.

\section{Referencias}

Aguilar, G., Bonilla J., Cubero L., Jiménez, C., Murillo, A., Montenegro, F., Mora, O., Núñez, R., Ramirez, A., Rojas, L., Qirós,M. y Vega, F. 2012. La Educación Especial en Costa Rica: antecedentes, evolución, nuevas tendencias y desafíos. Costa Rica: Centro Nacional de Recursos para la Educación Inclusiva (CENAREC), 1 ed. - San José, C.R. : Procesos Litográficos de Centroamérica.

Andújar, C., Benítez, N., Blanco, R., Bueno, J., Duk, C., Hernández, L. y Torres, D. 2014. Avances y desafios de la educación inclusiva en Iberoamérica. España: Metas Educativas 2021, Fundación MAPFRE.

Angulo, M. del C., Gijón, A., Luna, M. y Prieto I. 2015. Sindrome de Down: Manual de atención al alumnado con necesidades especificas de apoyo educativo derivadas de Sindrome de Down. España: Junta de Andalucía, Consejería de Educación.

Asamblea Legislativa. 2004. Ley 7600 Igualdad de Oportunidades para las Personas con Discapacidad. Costa Rica: Asamblea Legislativa de la República de Costa Rica. 
Barker, P. 2005. What is IEEE Learning Object Metadata/IMS Learning Resource Metadata? Reino Unido: Cetis Standards Briefings Series I(5), Joint Information Systems Committee (JISC) of the University of Boltonv.

Bravo, J., Hérvas R. y Chavira, G. 2005. Ubiquitous Computing in the Classroom: An Approach through Identification Process. España: Journal of Universal Computer Science. XI (9): 1494-1504.

Buckley, S. 2000. Living with Down syndrome. Reino Unido: Down Syndrome Education International.Down Syndrome.

Carpio, M. de los A. 2011. Eficacia de las estrategias pictofónicas en la enseñanza de la lectura inicial en Costa Rica: Un estudio longitudinal.Costa Rica: Revista Actualidades Investigativas en Educación XI (3): 1-33.

Cordero F. y García, J. 2014. Las TIC en la formación docente. Costa Rica: Congreso Iberoamericano de Ciencia, Tecnología, Innovación y Educación.

Cortez, A., Vega, H. y Pariona, J. 2009. Procesamiento de lenguaje natural. Perú: Revista de investigación de Sistemas e Informática de la Universidad Nacional Mayor de San Marcos, VI (2): 45-54.

Epstein, A. S., Hobmann, C. y Hobmann, M. 2002. How Young Children Learn to Read in High / Scope Programs. Estados Unidos: High/Scope Educational Research Foundation.

Escamilla, S. 1983. El Niño con Síndrome de Down. México: John Langdon Down Foundation, XLVIII (3): 335.

Ferreyra, J., Méndez, A. y Rodrigo, M. 2009. El uso de las TIC en la Educación Especial: Descripción de un Sistema Informático para Niños Discapacitados Visuales en Etapa Preescolar. España:
Revista Iberoamericana de Tecnología en Educación y Educación en Tecnología.

INEC Costa Rica. 2011. X Censo Nacional de Población y VI de Vivienda 2011: Características Sociales y Demográficas. Costa Rica: InstitutoNacional de Estadísticas y Censos.

Ishii, H. y Ullmer, B. 1997. Tangible bits. EstadosUnidos: Proceedings of the SIGCHI conference on Human factors in computing systems CHI 97. 39: 234-241.

Jadán-Guerrero, J., Guerrero, L., López, G., Cáliz, D., y Bravo, J. 2015. Creating TUIs Using RFID Sensors--A Case Study Based on the Literacy Process of Children with Down Syndrome. Suiza: Journal Sensors, $\mathrm{XV}(7)$.

Jadán-Guerrero, J., Jaen, J., Carpio, M. de los Á., y Guerrero, L. A. 2015. Kiteracy: A Kit of Tangible Objects to Strengthen Literacy Skills in Children with Down Syndrome. Estados Unidos: Proceedings in Interaction Design and Children Conference, IDC 2015: 15-18.

Jadán-Guerrero, J., López, G. y Guerrero, L. A. 2014. Use of Tangible Interfaces to Support a Literacy System in Children with Intellectual Disabilities. Irlanda: Proceedings of International Conference on Ubiquitous Computing \& Ambient Intelligence: $108-115$.

Kriara, L., Alsup, M., Corbellini, G., Trotter, M., Griffin, J. D. y Mangold, S. 2013. RFID shakables Pairing Radio-Frequency Identification Tags with the Help of Gesture Recognition. Estados Unidos: Proceedings of the ninth ACM conference on Emerging networking experiments and technologies - CoNEXT '13: 327-332.

Los Santos Aransay, A. 2009. Computación Ubicua: Trabajo Individual. España: Diseño de interacción centrada en el usuario, Universidad de Vigo: 1-10. 
Muñoz, L., Brenes, M., Bujanda, M., Mora, M., Núñez, O. y Zúñiga, M. 2013. Las políticas TIC en los sistemas educativos de América Latina: Caso Costa Rica. Costa Rica: Programa TIC y Educación Básica. UNICEF: 1-123.

Necuzzi, C. 2013. El desarrollo cognitivo involucrado en los procesos de aprendizaje $y$ enseñanza con integración de las TIC. Argentina: Programa TIC y Educación Básica. UNICEF: 1-158.

O’Malley, C., y Stanton-Fraser, D. 2004. Literature Review in Learning with Tangible Technologies. Reino Unido: Learning Sciences Research Institute, FutureLab at Nfer, University of Nottingh.

ONU.2006. Convención sobre los Derechos de las Personas con Discapacidad y su Protocolo Facultativo, Nueva York: Organización de la Naciones Unidas: 1-35.

Pennington, B., Moon, J., Edgin, J., Stedron, J., y Nadel, L. 2012. The neuropsychology of Down syndrome: evidence for hippocampal dysfunction. Estados Unidos: PubMed, LXXIV (1): 75-93.

Ramos, J. 2004. Enseñar a leer a los alumnos con discapacidad intelectual: una reflexión sobre la práctica. España: Revista Iberoamérica de Educación, 34: 201-216.

Rodríguez, E. 2004. Programa de educación emocional para niños y jóvenes con sindrome de Down. España: Revista Síndrome de Down, 21: 84-93.

Sternberg, R., y Sternberg, K. 1999. Cognitive Psychology $6^{\text {th }}$ Edition, Estados Umidos: Wadsworth, Cengage Learning: 15-17.
Troncoso, M., y Mercedes, D. 2009. Sindrome de Down: Lectura y Escritura. España: Fundación Cantabria.

UNESCO. 2008. La Educación Inclusiva: El Camino Hacia El Futuro. Ginebra: Conferencia Internacional de Educación, Organización de las Naciones Unidas para la educación, la ciencia y la cultura.

Vaillant, D. 2013.Integración de TIC en los sistemas de formación docente inicial y continua para la Educación Básica en América Latina. Argentina: Programa TIC y Educación Básica. UNICEF: 1-49.

Vygotski, L., Leóntiev, A., y Luria, A. 2004. Psicologia y Pedagogía. España: Ediciones AKAL, 99: 1-320.

Wall, D. W. 1980. Educación Constructiva para grupos especiales: Niños con problemas de aprendizaje. Ginebra: UNESCO: 1-156.

Weiser, M. 1991. The Computer for the 21 century. Estados Unidos: ACM SIGMOBILE Mobile Computing and Communications Review, III (3): 3-11.

Wisniewski, K. E., Wisniewski, H. M., y Wen, G. Y. 1984. Occurrence of Neuropathologd Changes and Dementia of Alzheimer's Disease. Estados Unidos: Annals of Neurology, XVII (3): 278-282.

Yang, Y., Conners, F., y Merrill, E. 2014. La habilidad visuo-espacial en el síndrome de Down: ¿es realmente un punto fuerte ?. España: Fundación Iberoamericana Down 21: 134-141.

\section{(c) $(\mathcal{B Y} \Theta \Theta$}

Este obra está bajo una licencia de Creative Commons Reconocimiento-NoComercial-SinObraDerivada 4.0 Internacional. 
